\title{
Bladder (ICRU) dose point does not predict urinary acute toxicity in adjuvant isolated vaginal vault high-dose-rate brachytherapy for intermediate-risk endometrial cancer
}

\author{
Lucas Gomes Sapienza, MD1,2, Antonio Aiza, MD², Maria José Leite Gomes, MD³, Michael Jenwei Chen, MD², \\ Antonio Cassio de Assis Pellizzon, MD, PhD², David B. Mansur, MD4, Glauco Baiocchi, MD, PhD² \\ 'Clínicas Oncológicas Integradas (COI), Rio de Janeiro, Brazil, ${ }^{2}$ A.C. Camargo Cancer Center, São Paulo, Brazil, ${ }^{3}$ Hospital Federal dos \\ Servidores do Estado do Rio de Janeiro (HFSE-RJ), Rio de Janeiro, Brazil, ${ }^{4}$ University Hospitals Seidman Cancer Center, Cleveland, OH, USA
}

\begin{abstract}
Purpose: High-dose-rate brachytherapy (HDR-BT) alone is an adjuvant treatment option for stage I intermediaterisk endometrial cancer after complete surgical resection. The aim of this study was to determine the value of the dose reported to ICRU bladder point in predicting acute urinary toxicity. Oncologic results are also presented.

Material and methods: One hundred twenty-six patients were treated with postoperative HDR-BT 24 Gy $(4 \times 6$ Gy) per ICRU guidelines for dose reporting. Cox analysis was used to identify variables that affected local control. The mean bladder point dose was examined for its ability to predict acute urinary toxicity.

Results: Two patients (1.6\%) developed grade 1 gastrointestinal toxicity and 12 patients (9.5\%) developed grades $1-2$ urinary toxicity. No grade 3 or greater toxicity was observed. The mean bladder point dose was $46.9 \%$ (11.256 Gy) and $49.8 \%(11.952 \mathrm{~Gy})$ for the asymptomatic and symptomatic groups, respectively $(p=0.69)$. After a median follow-up of 36.8 months, the 3-year local failure and 5-year cancer-specific and overall survival rates were 2.1\%, 100\%, and 94.6\%, respectively. No pelvic failure was seen in this cohort. Age over 60 years $(p=0.48)$, lymphatic invasion $(p=0.77)$, FIGO histological grade $(p=0.76)$, isthmus invasion $(p=0.68)$, and applicator type (cylinder $\times$ ovoid) $(p=0.82)$ did not significantly affect local control.

Conclusions: In this retrospective study, ICRU bladder point did not correlate with urinary toxicity. Four fractions of 6 Gy HDR-BT effected satisfactory local control, with acceptable urinary and gastrointestinal toxicity.

Key words: endometrial cancer, high-dose-rate brachytherapy, ICRU 38, urinary toxicity.

\section{Purpose}

Radiotherapy is an important adjuvant treatment option for intermediate-risk stage I endometrial cancer. Several phase III trials have shown that radiotherapy in intermediate-risk patients decreases locoregional recurrences but does not improve survival $[1,2,3,4,5,6,7,8,9,10]$. Regarding vaginal control, the Postoperative Radiation Therapy in Endometrial Cancer (PORTEC-2) trial concluded that isolated brachytherapy (BT) is equivalent to external beam radiotherapy (EBRT) for intermediate-risk patients [8]. Moreover, BT is associated with less gastrointestinal toxicity $[2,8,11]$.

Report number 38 by the International Commission on Radiation Units and Measurements (ICRU) established the technical basis for 2-dimensional intracavitary BT [12], and suggested that the dose to the bladder and rectum be reported using reference points, visualized on simple orthogonal X-rays. Recently, regarding vaginal vault treatment, the American Brachytherapy Society (ABS) also recommended considering the documentation of doses to the adjacent normal tissue, particularly of these 2 adjacent organs at risk (OARs) [13].

In low-dose-rate (LDR) BT, for example, Perez et al. demonstrated a lack of correlation between rectal point dose and the incidence of late grade 2 rectosigmoid toxicity, and between ICRU bladder point dose and grade 2 or 3 urinary toxicity [14]. No study has examined the clinical significance of ICRU points and their ability to predict toxicity in treatment with high-dose-rate (HDR) BT alone.

The main objective of this study was to report toxicity to the rectum and bladder, and evaluate the reliability of 
the dose to the bladder in predicting urinary toxicity with postoperative vaginal vault HDR-BT alone. Further, we analyzed local failure (LF), pelvic failure (PF), metastasis incidence (MI), and overall survival (OS) in a cohort of patients who were treated with BT for intermediate-risk stage I endometrial cancer.

\section{Material and methods}

\section{Eligibility criteria}

The study cohort comprised patients with endometrial cancer who were initially treated with complete surgical resection and staging; classified as having stage I, intermediate-risk disease, and subsequently treated between 2009 and 2013. This period was selected to avoid the need for pelvic external beam irradiation for intermediate-risk stage I endometrial cancer, because our institutional guidelines changed after publication of the PORTEC-2 study [8].

The intermediate-risk patients were divided in $2 \mathrm{sub}$ groups. The high-intermediate-risk (HIR) group was based on the PORTEC-2 eligibility criteria: age greater than 60 years and 2009 FIGO stage IB (invasion greater than $50 \%$ of the myometrium) with grade 1 or 2 disease or 2009 FIGO stage IA (invasion less or equal $50 \%$ of the myometrium) with grade 3, and stage IIA disease, any age (except grade 3 with greater than $50 \%$ myometrium invasion). The remaining patients were classified as low-intermediate-risk (LIR), excluding low-risk IA G1 (without adverse pathological findings, such as lymphatic invasion and isthmus involvement).

\section{Dosimetry}

Dosimetric data were obtained from the treatment planning system $\left(\right.$ Eclipse $^{\circledR}$, version 11.0, Varian, Palo Alto, CA, USA) using simple orthogonal X-ray images (Figure 1). Reference points for rectal and bladder doses were acquired per ICRU 38 guidelines [12]. Briefly, the point of reference for the rectal dose was determined in the lateral radiograph, $5 \mathrm{~mm}$ behind the posterior vaginal wall, posterior to the vaginal applicator. The bladder reference point was obtained using a Foley catheter with the balloon filled with $7 \mathrm{cc}$ of radio-opaque fluid. The catheter was pulled downward to bring the balloon against the urethra. On the lateral radiograph, the bladder reference point was identified on the posterior surface of the balloon, on an anterior-posterior line through the center of the balloon. On the anterior-posterior (AP) radiograph, the reference point was taken as the center of the balloon.

Per institutional protocols, all patients were treated with HDR-BT devices (GammaMed-Varian, Palo Alto, CA, USA), targeting the proximal $2 \mathrm{~cm}$ of the vaginal vault for vaginal cylinders or the vaginal vault for ovoids. Although we did not report such values, the $2 \mathrm{~cm}$-proximal vaginal target was intended to decrease the vaginal late toxicity rate. The dose was prescribed at a depth of $0.5 \mathrm{~cm}$
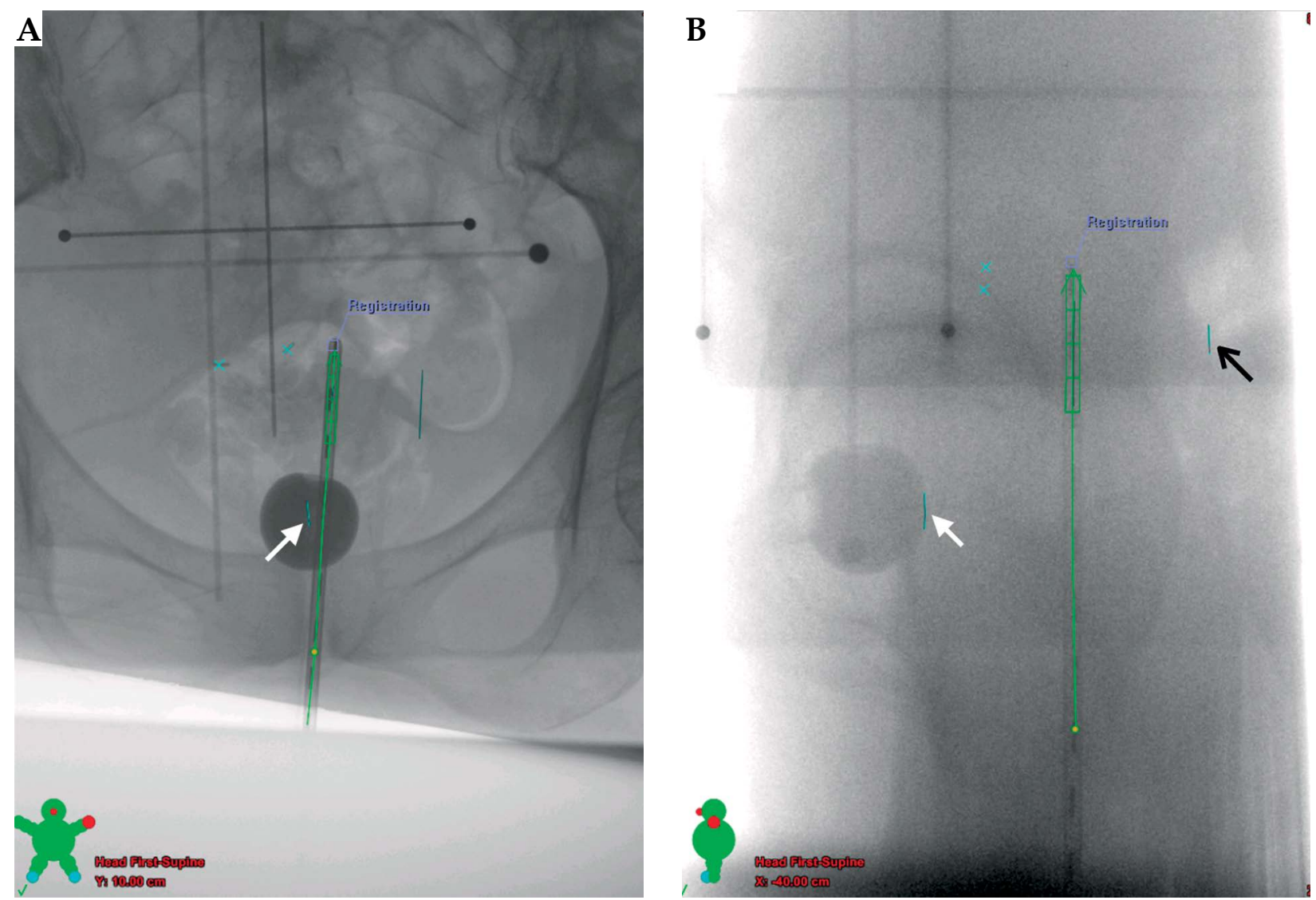

Fig. 1. Example of simple orthogonal X-ray images used for localization of vaginal cylinder and ICRU bladder (white arrow) and ICRU rectal reference points (black arrow) per the ICRU 38 report. A) Anteroposterior projection. B) Lateral projection 
from the mucosal surface. The dose was $24 \mathrm{~Gy}$, delivered in 4 weekly fractions of $6 \mathrm{~Gy}\left(\mathrm{BED}=72 \mathrm{~Gy}_{3} / 38.4 \mathrm{~Gy}_{10}\right)$ This regimen had an $\mathrm{EQD}_{2}$ dose of $32 \mathrm{~Gy}(\alpha / \beta$ ratio $=10)$.

\section{Toxicity scoring}

Acute toxicity was graded per the Common Terminology Criteria for Adverse Events (CTCAE v4.0) [15], and all patients were followed weekly during the treatment to assess acute toxicities. This study was limited to the evaluation of urinary and rectal toxicity. By student's $t$-test, we compared the mean doses of OARs between groups with (symptomatic group [SG]) and without urinary toxicity (asymptomatic group [AG]).

\section{Treatment outcomes}

Treatment outcomes were evaluated for patients with more than 1 year of follow-up. Survival times were calculated from the date of initiation of therapy (date of surgery). Pelvic lymph node recurrence alone or concurrent with local failure was considered PF. Local failure and survival analyses were performed by Kaplan-Meier method and log-rank test was used for the subgroup analysis. The institutional ethics research committee approved this study.

\section{Results}

One hundred twenty-six consecutive patients were analyzed. The median age at diagnosis was 61 years (range: 29-86). Seven patients were excluded from the analysis of oncological outcomes, because they were retrospectively considered to be high-risk $(n=6)$ or low-risk without adverse pathological findings $(n=1)$ but were included in the toxicity analysis. The median tumor size was $3.1 \mathrm{~cm}$ (range: $0.6-9.6 \mathrm{~cm}$ ). The patients and treatment profiles are detailed in Table 1. Brachytherapy treatments were delivered with a cylinder $(94.4 \%)$ or ovoids $(5.6 \%)$ at the discretion of the physician.

Two patients $(1.6 \%)$ developed grade 1 gastrointestinal (GI) toxicity and 12 patients $(9.5 \%)$ developed grades 1-2 urinary (GU) toxicity (Table 2). No grade 3 or greater GI or GU toxicity was observed. The mean bladder point dose was $46.9 \%$ of prescription (11.26 Gy) for the AG, and $49.8 \%$ of prescription (11.95 Gy) for the SG $(p=0.69)$. Of the 7 patients with grade 2 urinary toxicities, three $(42.8 \%)$ were also diagnosed with urinary tract infection, requiring treatment with oral antibiotics. As expected, the dose at the ICRU rectal point was $24 \mathrm{~Gy}$ in all cases, because the reference line for prescription $(0.5 \mathrm{~cm}$ depth from the mucosal surface) included the rectal reference point.

For patients with more than 1 year of follow-up $(n=104)$, after a median of 36.8 months, there were 2 vaginal failures (both at 18 months of follow-up) and 2 deaths, none that was related to progression of endometrial cancer $(1$ case of acute myocardial infarction and 1 case of sepsis after an attempt at surgical removal of an intestinal adhesion). The 3-year LF rate was 2.1\% (Figure 2A). The 5 -year cancer-specific survival rate was $100 \%$. As shown in Figure 2B, the 5-year OS was $94.6 \%$. No pelvic failure was observed in this cohort. One patient devel- oped distant metastasis to the lungs after 24 months of follow-up but remains alive after 1 year.

In a subgroup analysis, although both failures occurred in patients aged older than 60 years, age was not a statistically significant predictor of LF ( $p=0.48$ ) (Figure 2C). The IHR group was not associated with an increased risk of LF compared with intermediate-low-risk patients $(p=0.91)$ (Figure 2D). Similarly, lymphatic invasion $(p=0.77)$, FIGO histological grade $(p=0.76)$, isthmus invasion $(p=0.68)$, and applicator type (cylinder vs. ovoid) $(p=0.82)$ were not linked to LF (Figure 2C,D). Also,

Table 1. Patients and treatment characteristics

\begin{tabular}{|c|c|c|}
\hline Factors & $N$ & $\%$ \\
\hline \multicolumn{3}{|l|}{ Age } \\
\hline$<60 y$ & 54 & 42.9 \\
\hline$>60 y$ & 72 & 57.1 \\
\hline \multicolumn{3}{|c|}{ Lymphadenectomy } \\
\hline None & 18 & 14.3 \\
\hline Sampling* & 14 & 11.1 \\
\hline Systematic ${ }^{\star \star}$ & 79 & 62.7 \\
\hline Not available & 15 & 11.9 \\
\hline \multicolumn{3}{|l|}{ FIGO group } \\
\hline IAGI & 7 & 5.6 \\
\hline IAG2 & 46 & 36.5 \\
\hline IAG3 & 34 & 27 \\
\hline IBG1 & 14 & 11.1 \\
\hline IBG2 & 21 & 16.7 \\
\hline IBG3 & 3 & 2.4 \\
\hline$\| A G 2$ & 1 & 0.8 \\
\hline \multicolumn{3}{|c|}{ Isthmus involvement } \\
\hline Negative & 107 & 84.9 \\
\hline Positive & 19 & 15.1 \\
\hline \multicolumn{3}{|c|}{ Angiolimphatic invasion } \\
\hline Negative & 118 & 93.7 \\
\hline Positive & 8 & 6.3 \\
\hline
\end{tabular}

*Sampling: less than 12 lymph nodes removed. **Systematic: 12 or more lymph nodes removed. FIGO 2009 staging

Table 2. Toxicity

\begin{tabular}{ccccc} 
Grade & $\mathrm{Gl}(n, \%)$ & $\mathrm{GU}(n, \%)$ & $\begin{array}{c}\text { Positive urine } \\
\text { culture }(n)\end{array}$ & $\begin{array}{c}\text { Positive urine } \\
\text { culture }(\%)\end{array}$ \\
\hline 1 & $2(1.6)$ & $5(4)$ & 0 & 0 \\
\hline 2 & 0 & $7(5.5)$ & 3 & 42.8 \\
\hline $3-5$ & 0 & 0 & 0 & 0
\end{tabular}

GI - gastro-intestinal, GU - genito-urinary 
A

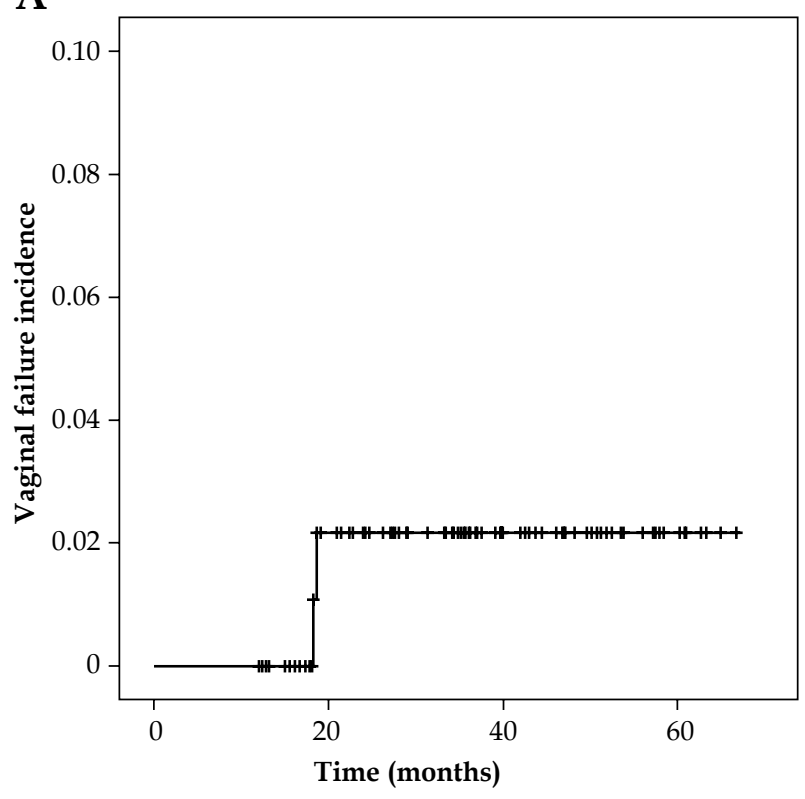

C

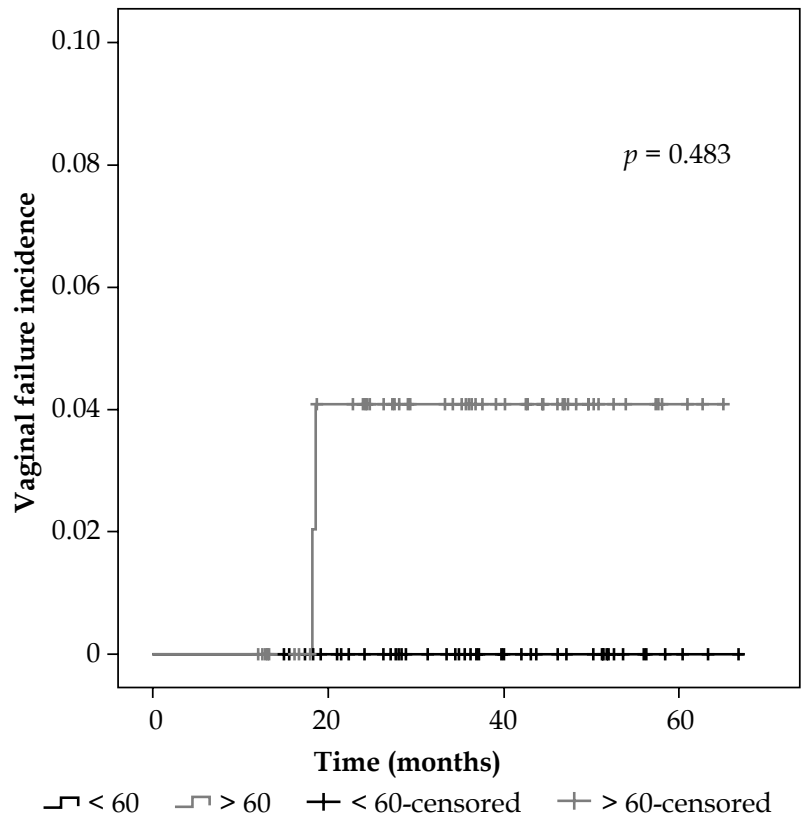

B

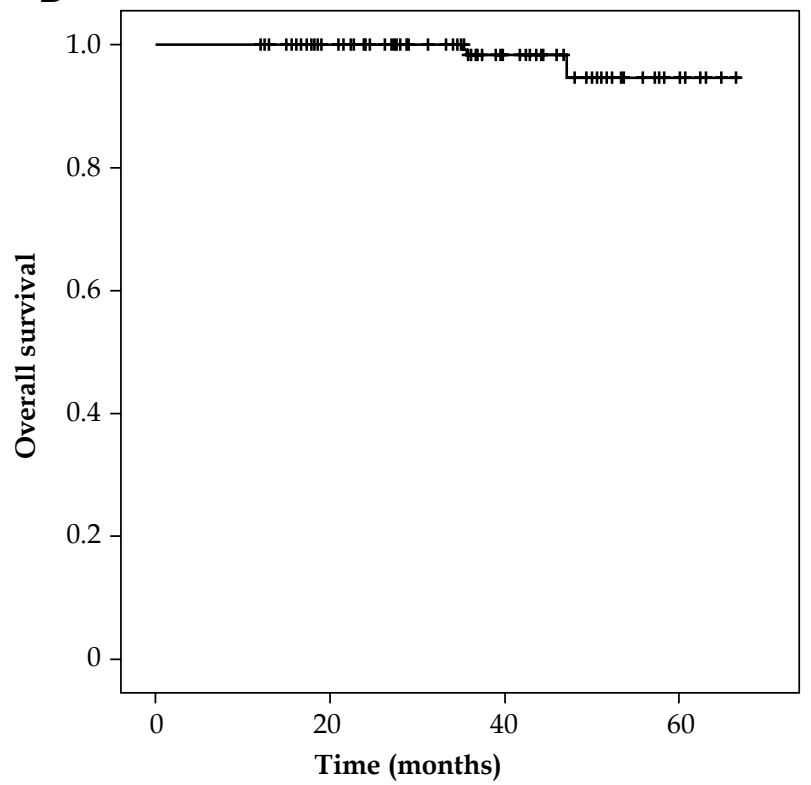

D

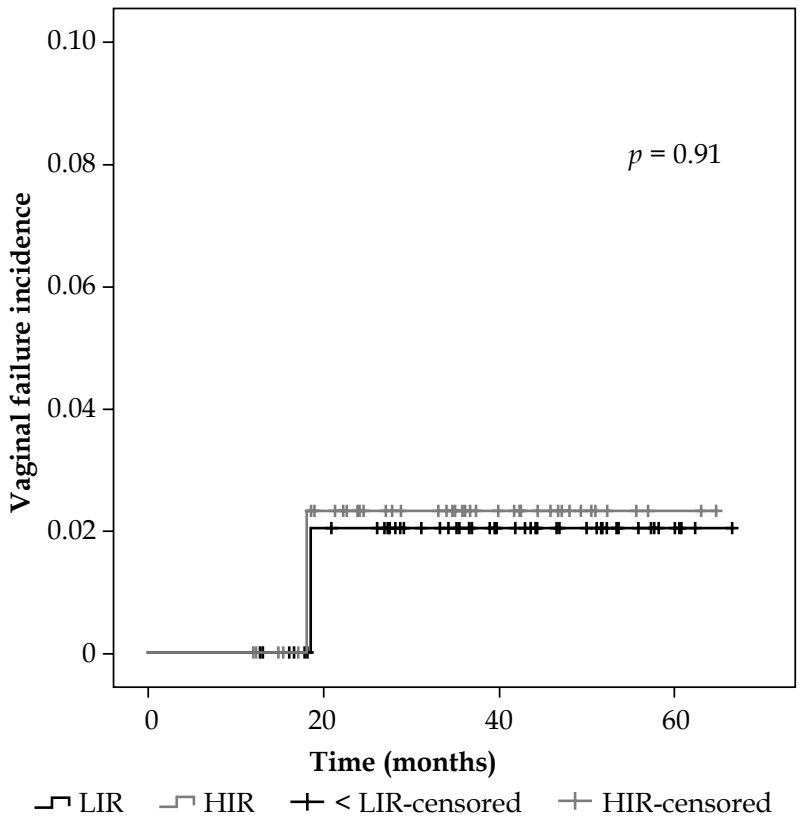

Fig. 2. Kaplan-Meier survival tables. A) Incidence of vaginal failure. B) Overall survival. C) Incidence of vaginal failure as a function of age. D) Incidence of vaginal failure as a function of risk. LIR - low-intermediate-risk; HIR - high-intermediate-risk

lymphadenectomy failed to correlate with differences in local recurrence $(p=0.43)$ and OS - the 5-year OS rates were $95.5 \%$ and $94.7 \%$ with and without lymphadenectomy, respectively $(p=0.44)$.

\section{Discussion}

The small number of events 3 disease failures (2 LF and 1 distant metastasis) and 14 treatment G1-G2-related adverse effects] in this cohort demonstrates the outstanding prognosis and safety profile of using BT alone in the adjuvant treatment of stage I intermediate-risk endometrial cancer patients.
In this study, $1.6 \%$ of patients developed acute G1-G2 GI toxicity, which is favorable compared with the vaginal brachytherapy-alone arm in PORTEC-2 [8], which reported a $12.6 \%$ acute GI-G2 GI toxicity rate. This excellent toxicity profile, even with the higher $\mathrm{EQD}_{2}$ dose in our regimen - 32 Gy vs. 29.8 Gy in PORTEC-2 - can be explained in part by the activation of sources at only $2 \mathrm{~cm}$ of the vaginal vault. Because our study did not evaluate vaginal toxicity, and because PORTEC-2 did not present acute GU toxicity, these adverse effects were not compared.

ICRU classically establishes surrogate points for the assessment of toxicity, based on 2-dimensional references [12]. The principal criticism of these points is the lack 
of a proven correlation between the reference point for calculation of the dose and clinical toxicity. This finding might be due to technical inaccuracies in the dose measurements, considering that these points are bidimensional representations of a 3-dimensional distribution of dose. There are differences between the ICRU bladder dose and the volumetric $(0.1 \mathrm{cc}, 1 \mathrm{cc}$, and $2 \mathrm{cc})$ or maximum dose to this organ [16]. Also, the recommendation by the ICRU to pull down the catheter to place the balloon against the urethra is one example of a potential source of inaccuracy [12]. The pressure that is used can generate variable displacement of the reference point, because this is related to the position of the balloon, which might fail to be accompanied by equal displacement of the bladder.

In addition to the technical uncertainties of vaginal cuff brachytherapy alone, the exact origin of acute symptoms is unknown. The urinary pain that is associated with therapeutic radiation might be related to the dose to the entire bladder, part of the organ, trigone of bladder [17], and the urethra [18]. As noted in our study, the urine analysis diagnosed an infection in over $40 \%$ of cases with grade 2 urinary toxicity, likely due to bladder catheterization, a possible explanation for urinary pain. This finding suggests another limitation of the ICRU dose reporting system, which requires the presence of the urinary catheter balloon inside of the bladder to estimate its position [12]. Similarly, there might be a causative relationship between the dose to the rectum, sigmoid [19], individual loops of the small bowel, and the intestinal bag and gastrointestinal symptoms but the limited number of gastrointestinal toxicity events made it impossible to draw any conclusions about the value of the ICRU rectal point.

Conversely, planning with computed tomographyassisted brachytherapy can guide individualized treatment of the vagina with better coverage of any tissue behind the mucosa that is inaccessible by physical examination or simple $\mathrm{x}$-ray, and predict the dose to OARs, possibly improving the toxicity profile $[20,21]$. Although the dosimetric differences between ICRU point data and the maximum doses by volumetric analysis using ultrasonography [22] or computerized tomography [23] are known, no study has demonstrated the superiority of these image methods over the evaluation of simple orthogonal x-ray reference points. To this end, a recently opened trial (NCT 02091050) is recruiting patients who have undergone hysterectomy for gynecological neoplasms to compare the accuracy of 2D vs. 3D planning with regard to the ability to predict toxicity.

All patients were homogeneously treated with adjuvant brachytherapy, and although lymphadenectomy was performed at the discretion of the surgeon, $60 \%$ of subjects underwent systematic pelvic lymphadenectomy. However, the extent of lymph node resection did not influence their outcomes, as suggested by the results of other more powered studies [24, 25]. This rate of lymphadenectomy, in turn, is more favorable than the PORTEC-2 trial [8], in which the incidence of pelvic failure was $3.8 \%$ in patients who did not undergo lymph node resection and were randomized to the no-pelvic radiation treatment arm.
Regarding the oncological results, our study is one of the largest experiences with HDR BT, using a fractionation of $4 \times 6 \mathrm{~Gy}$, and demonstrating equivalent results with over $97 \%$ vaginal control in 3 years, as the PORTEC-2 trial [8]. Excellent local control for low-risk endometrial cancer was achieved by Petereit et al. [26], using 2 insertions of 16.2 Gy each, prescribed to the vaginal surface. Similar results have been published, with over $95 \%$ vaginal vault local control in intermediate-risk cases using 5 fractions of 5.5 Gy [27] and 3 fractions of 7 Gy $[28,29]$. Because most local recurrences occur during the first 2 years of follow-up, our median follow-up time of 37 months appears to be adequate for evaluating local recurrences in this retrospective cohort.

\section{Conclusions}

A favorable toxicity profile (less than $10 \%$ grade $1-2$ acute reactions) could not be predicted by the ICRU 38 urinary reference point. The reliability of the ICRU 38 urinary reference point in this setting should be reevaluated in future trials. HDR BT alone with 4 fractions of $6 \mathrm{~Gy}$ is an adequate fractionation schedule for adjuvant treatment of intermediate-risk (high or low) stage I endometrial cancer in terms of local control.

\section{Acknowledgments}

We thank Dr. Roberto Sakuraba for assistance in producing the images of this article.

\section{Disclosure}

Authors report no conflict of interest.

\section{References}

1. Aalders J, Abeler V, Kolstad P et al. Postoperative external irradiation and prognostic parameters in stage I endometrial carcinoma - clinical and histopatologic study of 540 patients. Obstet Gynecol 1980; 56: 419-427.

2. Onsrud M, Cvancarova M, Hellebust TP et al. Long-term outcomes after pelvic radiation for early stage endometrial cancer. J Clin Oncol 2013; 31: 3951-3956.

3. Creutzberg CL, van Putten WLJ, Koper PC et al. Surgery and postoperative radiotherapy versus surgery alone for patients with stage-1 endometrial carcinoma: multicentre randomised trial. Lancet 2000; 355: 1404-1411.

4. Scholten AN, van Putten WLJ, Beerman $\mathrm{H}$ et al. Postoperative radiotherapy for stage 1 endometrial carcinoma: longterm outcome of the randomized PORTEC trial with central pathology review. Int J Radiat Oncol Biol Phys 2005; 63: 834838 .

5. Creutzberg CL, Nout RA, Lybeert ML et al. Fifteen-year radiotherapy outcomes of the randomized PORTEC-1 trial for endometrial carcinoma. Int J Radiat Oncol Biol Phys 2011; 81: e631-638.

6. Keys HM, Roberts JA, Brunetto VL et al. A phase III trial of surgery with or without adjunctive external pelvic radiation therapy in intermediate risk endometrial adenocarcinoma: a Gynecologic Oncology Group study. Gynecol Oncol 2004; 92: 744-751.

7. ASTEC/EN.5 Study Group, Blake P, Swart AM, Orton J et al. Adjuvant external beam radiotherapy in the treatment of endometrial cancer (MRC ASTEC and NCIC CTG EN.5 ran- 
domised trials): pooled trial results, systematic review, and meta-analysis. Lancet 2009; 373: 137-146.

8. Nout RA, Smit VT, Putter H et al. Vaginal brachytherapy versus pelvic external beam radiotherapy for patients with endometrial cancer of high-intermediate risk (PORTEC-2): an open-label, non-inferiority, randomised trial. Lancet 2010; 375: 816-823.

9. Harkenrider MM, Block AM, Siddiqui ZA et al. The role of vaginal cuff brachytherapy in endometrial cancer. Gynecol Oncol 2015; 136: 365-372.

10. Kellas-Ślęczka S, Wojcieszek P, Białas B. Adjuvant vaginal brachytherapy as a part of management in early endometrial cancer. J Contemp Brachytherapy 2012; 4: 247-252.

11. Nout RA, van de Poll-Franse LV, Lybeert ML et al. Longterm outcome and quality of life of patients with endometrial carcinoma treated with or without pelvic radiotherapy in the post operative radiation therapy in endometrial carcinoma 1 (PORTEC-1) trial. J Clin Oncol 2011; 29: 1692-1700.

12. Dose and Volume Specification for Reporting Intracavitary Therapy in Gynecology. ICRU Report No 38. 1985. http:// www.icru.org/home/reports/dose-and-volume-specification-for-reporting-intracavitary-therapy-in-gynecology-report-38

13. Small W, Beriwal S, Demanes DJ et al. American Brachytherapy Society consensus guidelines for adjuvant vaginal cuff brachytherapy after hysterectomy. Brachytherapy 2012; 11: 58-67.

14. Perez CA, Grigsby PW, Lockett MA et al. Radiation therapy morbidity in carcinoma of the uterine cervix: dosimetric and clinical correlation. Int J Radiat Oncol Biol Phys 1999; 44: 855-866.

15. Common Terminology Criteria for Adverse Events (CTCAE). Version 4.0. 2009. v4.03: June 14,2010). U.S. Department of Health and Human Services - National Institutes of Health. National Cancer Institute. http://evs.nci.nih.gov/ftp1/CTCAE/CTCAE_4.03_2010-06-14_QuickReference_5x7.pdf

16. Madan R, Pathy S, Subramani V et al. Comparative evaluation of two-dimensional radiography and three dimensional computed tomography based dose-volume parameters for high-dose-rate intracavitary brachytherapy of cervical cancer: a prospective study. Asian Pac J Cancer Prev 2014; 15: 4717-4721.

17. Ghadjar P, Zelefsky MJ, Spratt DE et al. Impact of dose to the bladder trigone on long-term urinary function after highdose intensity modulated radiation therapy for localized prostate cancer. Int J Radiat Oncol Biol Phys 2014; 88: 339-344.

18. Ishiyama $\mathrm{H}$, Kitano $M$, Satoh $\mathrm{T}$ et al. Genitourinary toxicity after high-dose-rate (HDR) brachytherapy combined with Hypofractionated External beam radiotherapy for localized prostate cancer: an analysis to determine the correlation between dose-volume histogram parameters in HDR brachytherapy and severity of toxicity. Int J Radiat Oncol Biol Phys 2009; 75: 23-28.

19. Al-Booz H, Boiangiu I, Appleby H et al. Sigmoid colon is an unexpected organ at risk in brachytherapy for cervical cancer. J Egypt Nat Canc Inst 2006; 18: 156-160.

20. Holloway CL, Macklin EA, Cormack RA et al. Should the organs at risk be contoured in vaginal cuff brachytherapy? Brachytherapy 2011; 10: 313-317.

21. Iatì $G$, Pontoriero $A$, Mondello $S$ et al. Three-dimensional treatment planning for vaginal cuff brachytherapy: Dosimetric effects on organ at risk according to patients position. Brachytherapy 2014; 13: 568-571.

22. Barillot I, Horiot JC, Maingon P et al. Maximum and mean bladder dose defined from ultrasonography: Comparison with ICRU reference in gynaecological brachytherapy. Radiother Oncol 1994; 30: 231-238.
23. Kapp KS, Stuecklschweiger GF, Kapp DS et al. Dosimetry of intracavitary placements for uterine and cervical carcinoma: Results of orthogonal film, TLD, and CT-assisted techniques. Radiother Oncol 1992; 24: 137-146.

24. Benedetti Panici P, Basile S, Maneschi F et al. Systematic pelvic lymphadenectomy vs no lymphadenectomy in early-stage endometrial carcinoma: randomized clinical trial. I Natl Cancer Inst 2008; 100: 1707-1716.

25. ASTEC study group, Kitchener H, Swart AM, Qian $Q$ et al. Efficacy of systematic pelvic lymphadenectomy in endometrial cancer (MRC ASTEC trial): a randomized study. Lancet 2009; 373: 125-136.

26. Petereit DG, Tennehill SP, Grosen EA et al. Outpatient vaginal cuff brachytherapy for endometrial cancer. Int J Gynecol Cancer 1999; 9: 456-462.

27. Atahan IL, Ozyar E, Yildiz F et al. Vaginal high dose rate brachytherapy alone in patients with intermediate- to high-risk stage I endometrial carcinoma after radical surgery. Int J Gynecol Cancer 2008; 18: 1294-1299.

28. Cengiz M, Singh AK, Grigsby PW. Postoperative vaginal brachytherapy alone is the treatment of choice for grade 1-2, stage IC endometrial cancer. Int J Gynecol Cancer 2005; 15: 926-931.

29. Eldredge-Hindy HB, Eastwick G, Anne PR et al. Adjuvant vaginal cuff brachytherapy for high-risk, early stage endometrial cancer. J Contemp Brachytherapy 2014; 6: 262-270. 